\title{
Radium-223 dichloride: illustrating the benefits of a multidisciplinary approach for patients with metastatic castration-resistant prostate cancer
}

This article was published in the following Dove Press journal:

Journal of Multidisciplinary Healthcare

5 June 2015

Number of times this article has been viewed

\author{
Joseph F Renzulli II \\ Jennifer Collins \\ Anthony Mega \\ Genitourinary Multidisciplinary \\ Clinic, The Miriam Hospital, \\ Providence, RI, USA
}

Correspondence: Joseph F Renzulli II Genitourinary Multidisciplinary Clinic, The Miriam Hospital, 195 Collyer Street, Suite 20I, Providence, RI 02904, USA

$\mathrm{Tel}+$ I 40I 2727799

Fax + I 40I 4539078

Email jrenzulli@lifespan.org

\begin{abstract}
Improving options for patients with metastatic castration-resistant prostate cancer (mCRPC) provide latitude in designing treatment plans that meet patients' medical needs and personal goals. The field's rapid evolution opens avenues for contributions by multiple medical specialties and requires considering more options to ensure that each patient receives the most appropriate care. A multidisciplinary clinic (MDC) focusing on patients with cancers of the genitourinary tract demonstrates an efficient and cost-effective means of integrating the diverse professional knowledge and skills needed to develop an optimal patient treatment plan. As a guide to establishing an MDC for patients with MCRPC, this article describes the operation of the Genitourinary MDC at The Miriam Hospital in Providence, RI - specifically, the successful incorporation of radium-223 dichloride (radium-223) into the treatment algorithm for men with mCRPC and symptomatic bone metastases. Radium-223 is a new treatment that, unlike earlier radionuclide therapies, has shown a survival advantage in a large randomized phase 3 trial (ALSYMPCA). The overall survival benefit was comparable to that of newer immunoand hormonal therapies in similar populations. Radium-223 treatment also delayed onset of symptomatic skeletal events. Both benefits were independent of prior docetaxel therapy or concurrent bisphosphonate use. In our clinic, radium-223 is used primarily to extend patient survival. Patient selection, patient management, and treatment sequencing are discussed here in the context of a multidisciplinary environment.
\end{abstract}

Keywords: radium-223 dichloride, prostate cancer, castration-resistant prostate cancer, multidisciplinary clinic, best practices

\section{Introduction}

Docetaxel was approved in the USA for the treatment of patients with metastatic castration-resistant prostate cancer (mCRPC) more than a decade ago. Until recently, there were no significant improvements in treatment options for men with mCRPC. Advances in molecular oncology and targeted therapies have revolutionized the treatment of many other advanced solid tumors and hematologic malignancies. Since 2010, we have enjoyed the approval of sipuleucel-T immunotherapy, ${ }^{1}$ cabazitaxel chemotherapy, ${ }^{2}$ and oral agents abiraterone acetate ${ }^{3}$ and enzalutamide ${ }^{4}$ and the recent approval of the first-in-class alpha-emitting radionuclide radium-223 dichloride (radium-223). ${ }^{5}$ The treatment options have expanded dramatically, resulting in longer survival and improved quality of life for our patients with mCRPC.

Accompanying this treatment evolution is an increase in treatment choice, in that now each patient's disease can be managed individually. However, controversies have arisen around questions of patient selection, combining treatments with other 
modalities, and optimal treatment sequencing, including identifying molecular biomarkers to help guide sequencing. For these reasons, treatment of patients with advanced prostate cancer increasingly requires both a specialized focus on this disease and the collaboration of committed urologists, medical oncologists, radiation oncologists, nuclear medicine physicians, and essential ancillary support services in a structured patient-focused multidisciplinary setting.

\section{The Genitourinary Multidisciplinary Clinic}

The Genitourinary Multidisciplinary Clinic (MDC) was opened in 2007 in an effort to mainstream the care of patients with prostate cancer at the The Miriam Hospital in Providence, RI. The Miriam Hospital is affiliated to the Warren Alpert School of Medicine, Brown University. The MDC has cared for more than 22,000 patients with prostate cancer. Radium-223 is an ideal example of a drug therapy for which multidisciplinary interaction best integrates treatment and optimizes patient outcome. Our clinic's incorporation of radium-223 treatment illustrates the benefit of an efficient and cost-effective workflow in a multidisciplinary environment.

In the MDC, four medical disciplines are involved: medical oncology, radiation oncology, urologic oncology, and nuclear medicine, with support from other services to enhance the patient experience and operational efficiency. This dynamic group extends service beyond the medical specialties: psychiatric service is provided for newly diagnosed patients with need, the counsel of a licensed nutritionist is available, a nurse navigator assists in the treatment management process, and a nurse practitioner assists in patient management. Sexual and continence therapy and the assistance of a social worker are also available as needed (Figure 1).

Patients are typically referred to the MDC either by a urologist recognizing the need for the comprehensive expertise of the MDC, by a primary care physician following a new diagnosis or for a second opinion, or by other subspecialties for a second opinion after referral from an outside urologist. Patients are seen within 2 weeks of referral to reduce the period of anxiety for the patient and family members. Before patients are seen, records are obtained and reviewed, imaging is gathered, and a genitourinary pathologist reviews tissue sections. It is critical to have all pathology reviewed, as there is significant interobserver variability in the evaluation of prostate cancer pathology on biopsy specimens, which could make the difference between active surveillance and active therapy in patients.

An important aspect of the MDC process is the initial consultation. Radiologic films and pathology reports are reviewed with the patient and family members, and the initial treatment plan is developed. Counseling regarding the patient's quality of life expectations and important goals is useful at this time in formulating the best individual treatment plan. ${ }^{6}$ The meeting includes physicians representing

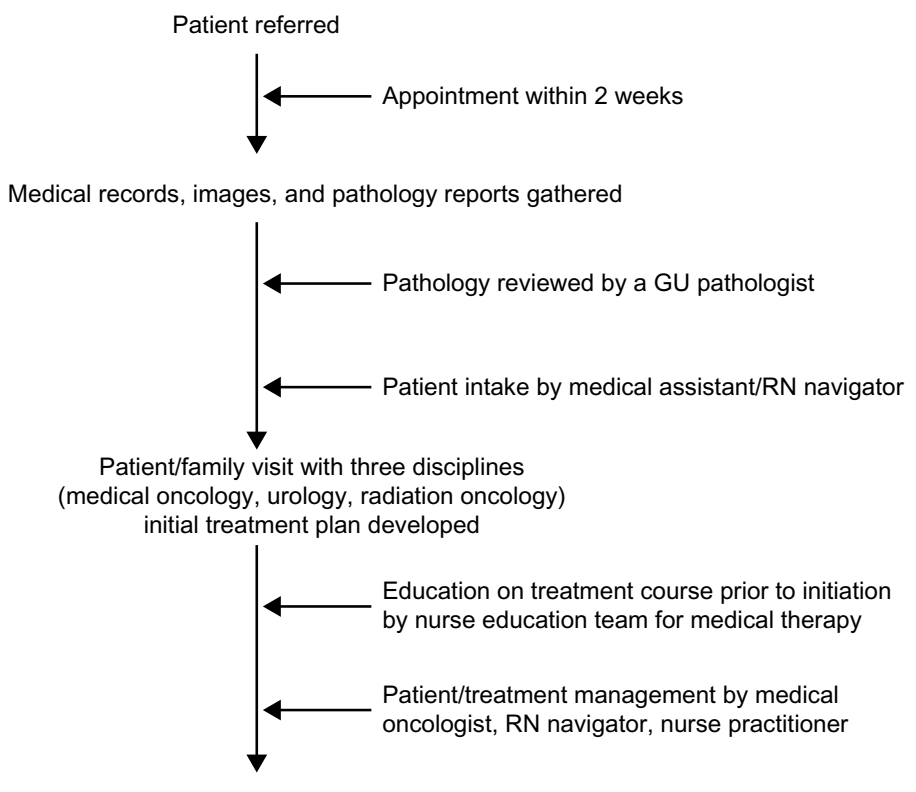

Nutritionist, social work, psychology input as needed

Figure I Flow of patients with mCRPC treated at the The Miriam Hospital MDC.

Abbreviations: mCRPC, metastatic castration-resistant prostate cancer; MDC, multidisciplinary clinic; GU, genitourinary; RN, registered nurse. 
each medical specialty and involves the patient and family in real-time discussion of available, appropriate management options. Synchronous counseling avoids a series of separate meetings and reduces patient anxiety, ensures a consensus regarding the treatment plan, and reassures the patient that all appropriate options have been properly discussed and considered with respect to him as an individual. It also controls the intrinsic biases that physicians may present to the patient in a one-on-one environment.

If the patient undergoes surgical intervention, he typically returns to the MDC for a postoperative pathology review and discussion with the team regarding the possible need for adjuvant therapies, such as radiation therapy, hormonal deprivation, or chemotherapy. The patient may remain in the MDC for follow-up, especially if he is considered at high risk for disease recurrence. In the case of a favorable prognosis, the patient usually returns to the primary referring urologist for follow-up. If a biochemical recurrence occurs or metastasis develops - as may be indicated by a rising prostate-specific antigen (PSA) level, changes in other laboratory values, pathology on imaging, or an increase in disease symptoms - the patient may be seen as a repeat referral for discussion of additional therapy.

Outside a multidisciplinary setting such as the MDC, radiation oncologists and nuclear medicine physicians rarely encounter a patient with $\mathrm{mCRPC}$, unless referred by a urologist or a medical oncologist. The MDC setting allows these specialists to provide clinical input into the management plan. The nuclear medicine physician is involved in decisions about radium-223 treatment plans to ensure that patients meet criteria for safe administration of radium-223. This is a new role for nuclear medicine, in that radium-223 is the first radionuclide with demonstrated survival benefit ${ }^{5}$ for treatment of mCRPC beyond pain palliation. ${ }^{7}$

If bone metastases are present and patients are symptomatic (ie, on opioid or nonopioid analgesics or having received external beam radiation therapy [EBRT] within the previous 12 weeks), ${ }^{5}$ radium-223 is considered and the patient is treated in the nuclear medicine facility at The Miriam Hospital. Before initiating therapy, the patient is educated by a unique nurse education team, which provides information on the risks and benefits of radium-223 therapy, reviews treatment schedules and special precautions, and most importantly, answers patientinitiated questions about this first-in-class therapy. We have observed that nurse-guided treatment education reduces patient anxiety. ${ }^{8}$ The medical oncology team monitors the laboratory values before each treatment cycle and communicates with nuclear medicine in assessing the appropriateness of continuing therapy. Laboratory values are assessed before the initiation of radium-223 and before each subsequent cycle. A complete blood count, including platelets, is checked in the third week of the cycle, and transfusions are utilized if necessary to continue therapy. PSA and alkaline phosphatase are evaluated after the third cycle; however, if a patient is symptomatically improving, therapy continues regardless of the PSA value. The patient's pain scores, Eastern Cooperative Oncology Group status, and overall quality of life while on treatment are also assessed. During this time, the patient may also be receiving other medications, such as agents for hormonal deprivation and maintenance of bone health, both of which are overseen by the medical oncologist. Through this collaborative effort, a care plan to optimize delivery of this therapy is created, and initial outcomes can be monitored.

\section{Radium-223 therapy}

Radium-223 is approved for treatment of patients with CRPC with symptomatic bone metastases and no known visceral metastases. ${ }^{9}$ The recommended dosing regimen is six cycles of $50 \mathrm{kBq} / \mathrm{kg}$ radium-223 therapy 4 weeks apart. As a calcium mimetic, radium-223 binds to hydroxyapatite at sites of osteogenesis associated with metastatic prostate cancer. Radium-223 decays through emission of four highenergy alpha particles over short distances, resulting in predominantly irreparable double-stranded DNA breaks in nearby cells that lead to cell death. The short range of alpha particles ensures that nearby cells (tumor) are affected to a greater extent than more distant (normal) tissues.

It is important to differentiate alpha particle therapy with radium-223 from palliative therapy for bone pain with beta particle-emitting strontium-89 and samarium-153, beginning with an understanding of the physical and biological differences between alpha and beta particles. An alpha particle is a positively charged helium nucleus containing two protons and two neutrons, whereas a beta particle is a negatively charged electron; the differences in mass cover orders of magnitude. Compared with strontium-89, radium-223 deposits, on average, greater than 45-fold more energy over a 24 -fold shorter range, creating an area of bone metastases destruction that is both small and intense, with less damage to bone marrow (Table 1). ${ }^{10-13}$ Most important, patients treated with radium-223 survive significantly longer than those receiving placebo, whereas improvement in overall survival has not been confirmed in patients receiving strontium- 89 or samarium-153..$^{5,714}$ In the randomized phase 3 radium-223 ALSYMPCA study of 921 patients with symptomatic mCRPC and two or more bone metastases, radium-223 significantly improved survival compared with placebo 
Table I Key properties of radiopharmaceuticals used in treating bone metastases in patients with mCRPC

\begin{tabular}{lllllll}
\hline Radionuclide & $\begin{array}{l}\text { Half-life } \\
\text { (days) }\end{array}$ & $\begin{array}{l}\text { Principal } \\
\text { emission }\end{array}$ & $\begin{array}{l}\text { Average energy } \\
\mathbf{( M e V})\end{array}$ & $\begin{array}{l}\text { Average tissue } \\
\text { penetration } \\
(\mathbf{m m})\end{array}$ & DNA damage & $\begin{array}{l}\text { Bone:marrow } \\
\text { ratio }\end{array}$ \\
\hline Strontium-89 & 50.5 & $\beta$ & 0.58 & 2.4 & Single-stranded breaks & $1.6^{\mathrm{a}}$ \\
Samarium-153 & 1.9 & $\beta$ & 0.22 & 0.55 & Single-stranded breaks & $4.4^{\mathrm{b}}$ \\
Radium-223 & 11.4 & $\alpha$ & $27.4^{\mathrm{c}}$ & $<0.1$ & Double-stranded breaks & $10.3^{\mathrm{d}}$ \\
\hline
\end{tabular}

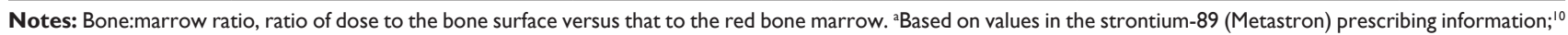
based on values in the samarium- I53 (Quadramet) prescribing information;" 'includes daughter nuclides; and destimate based on ICRP publication 67. ${ }^{2}$

Adapted from Clin Cancer Res, 2006, Volumel2(20 pt 2), Pages 6250s-6257s, Bruland OS, Nilsson S, Fisher DR, Larsen RH, High-linear energy transfer irradiation targeted to skeletal metastases by the alpha-emitter 223Ra: adjuvant or alternative to conventional modalities?, with permission from AACR. ${ }^{13}$

Abbreviations: $\mathrm{mCRPC}$, metastatic castration-resistant prostate cancer; ICRP, International Commission on Radiological Protection.

(median, 14.9 months vs 11.3 months; hazard ratio $=0.70$; 95\% CI, 0.58-0.83; $P<0.001)$ when either was administered with the best standard of care (eg, EBRT or treatment with glucocorticoids, antiandrogens, ketoconazole, or estrogens such as diethylstilbestrol or estramustine). ${ }^{5}$ Symptomatic disease was defined as regular use of analgesic medication, including nonsteroidal anti-inflammatory drugs or opioids, or treatment with EBRT for cancer-related bone pain within the previous 12 weeks. The increase in survival with radium-223 is comparable to that reported for other recently approved therapies - sipuleucel-T, ${ }^{1}$ enzalutamide, ${ }^{4}$ and abiraterone acetate $^{3}$ - in similar populations. In contrast, survival benefit has not been confirmed in a randomized study of strontium- 89 or samarium-153 as a single agent in patients with mCRPC and bone metastases. A Canadian multicenter randomized phase 3 trial comparing single injections of strontium- 89 (400 MBq) vs placebo as an adjunct to local EBRT in 126 patients with $\mathrm{mCRPC}$ and bone metastases reported benefits with strontium-89 in reducing progression of pain but no difference in overall survival. ${ }^{15} \mathrm{~A}$ randomized phase 3 EORTC study compared a single injection of strontium-89 (150 MBq) versus palliative EBRT in 101 patients with $\mathrm{mCRPC}$ and bone metastases. No significant difference in progressionfree survival was found, with borderline significantly longer overall survival $(P=0.0457)$ in patients receiving EBRT. ${ }^{16}$ Another trial comparing EBRT (focal or hemibody) versus strontium-89 $(200 \mathrm{MBq})$ in patients with $\mathrm{mCRPC}$ and bone metastases also reported effective pain relief in both treatment groups but no difference in overall survival. ${ }^{17}$ These singledose trials with strontium- 89 are not directly comparable to the multidose ALSYMPCA study, but they were conducted in very similar populations and represent the only randomized single-agent studies with strontium-89. No such studies with samarium-153 have been reported. ${ }^{7,14}$

The value of radium-223 therapy for patients with mCRPC and bone metastases has been recognized by regulatory authorities in the USA, Europe, and many other countries, whereas regulatory approvals for strontium-89 $\left(\right.$ Metastron $^{\circledR}$ ) and samarium-153 (Quadramet ${ }^{\circledR}$ ) specifically limit their use to relief of pain in patients with bone metastases. Further, National Comprehensive Cancer Network treatment guidelines for patients with prostate cancer recommend therapeutic use of radium-223 for patients with mCRPC and bone metastases with the highest level of confidence (level 1). No mention is made of palliative use; the guidelines specifically state that no survival advantage has been seen with any of the beta-emitting radionuclides, and they should be used only for palliation. ${ }^{18}$ Other organizations such as American Society of Clinical Oncology, ${ }^{19}$ American Urological Association, ${ }^{20}$ and European Society for Medical Oncology ${ }^{21,22}$ also recommend the use of radium-223 to treat bone metastases in patients with CRPC.

In the radium-223 ALSYMPCA study, patients receiving radium-223 also benefited, with a significantly prolonged time to onset of their first symptomatic skeletal event (median, 15.6 months vs 9.8 months; hazard ratio $=0.66$; $95 \%$ CI, $0.52-0.83 ; P<0.001)$ compared with those who received placebo. ${ }^{23}$ This treatment benefit and increased overall survival occurred independent of prior docetaxel therapy or concomitant bisphosphonate therapy. ${ }^{23}$ Consistent with our experience, radium-223 was associated with mild transient myelosuppression in ALSYMPCA ${ }^{5}$ and other trials. ${ }^{24-26}$ For patients who are particularly symptomatic, EBRT can be safely given with radium-223.27

While pain relief is an important proximate goal, the ultimate goal is to prolong survival and provide a good quality of life for patients. The use of radium-223 to target multifocal skeletal metastases, with the clinical benefit of prolonging time to symptomatic skeletal event and no excessive toxicity, is an effective tool in achieving this therapeutic goal.

\section{Patient selection}

Patient evaluation by the multidisciplinary team is key to successful treatment. The general patient selection criteria 
for radium-223 are listed in Table 2. Patients should have a life expectancy of at least 6 months and at least two sites of bone metastases, identified by technetium-99m-labeled phosphonate bone scan or ${ }^{18} \mathrm{~F}$-sodium fluoride positron emission tomography-computed tomography (PET/CT) scan, that are not suitable for treatment with EBRT. There should be no radiographic evidence of visceral metastases or bulky soft tissue metastases (brain, liver, lung, lymph nodes $>3 \mathrm{~cm}$ ) or evidence of chronic and/or active intestinal disease. Prior to initiating radium-223 therapy, patients should undergo whole-body CT scans to ensure appropriate eligibility.

Patients with mCRPC who complain of bone pain, regardless of its severity, are evaluated for radium-223 therapy. Pain managed with nonsteroidal anti-inflammatory drugs or over-the-counter analgesics such as acetaminophen may well indicate the presence of sufficient osseous metastatic disease to benefit from radium-223 therapy. This is especially the case in patients who have received abiraterone or enzalutamide, either before or after docetaxel therapy, and now have symptomatic or radiographic progression. Based on emerging data related to cross-resistance, ${ }^{28}$ there appears to be limited benefit in sequencing alternative endocrine therapies. The MDC may help facilitate such evidence-based decision making through a single disciplinary approach that may more easily guide the sequencing of oral agents for the given patient.

\section{Patient monitoring and follow-up}

During treatment, a nurse practitioner monitors laboratory values before each cycle and manages the liaison between nuclear medicine and medical oncology with the assistance of the nurse navigator. The mid-level practitioner is well informed about the appropriate monitoring of patients receiving radium-223 therapy and ensures that patients remain euvolemic throughout treatment. The nurse practitioner oversees continued supportive care and communicates any patient issues to the MDC team. Even with mid-level oversight, physicians remain involved in patient monitoring

Table 2 Selection criteria for patients with $\mathrm{mCRPC}$ to receive radium-223

I. At least two bone metastases identified by bone scan, not suitable for radiotherapy

2. Symptomatic disease - regular use of opioid or nonopioid analgesic medicine or recent EBRT for bone pain

3. No evidence of visceral metastases or bulky soft tissue metastases other than malignant lymphadenopathy $<3 \mathrm{~cm}$ in short-axis diameter

Abbreviations: $m C R P C$, metastatic castration-resistant prostate cancer; EBRT, external beam radiation therapy. and ultimately decide on whether to continue treatment or introduce other concurrent therapies, such as palliative radiation treatment.

\section{Discontinuing radium-223}

The recommended dosing regimen for radium-223 as per approved labeling is intended to achieve full survival benefit. Patients responding well to radium-223 therapy have their PSA values monitored every $2-3$ months, whereas earlier in the treatment, PSA levels may be checked more often; testing intervals are driven by the symptoms. Alkaline phosphatase levels are also typically monitored each cycle, as they may be a worthwhile biomarker in assessing response. A PSA level increase does not necessarily result in radium-223 discontinuation. A decision to discontinue radium-223 therapy prior to the completion of six cycles may be made for a patient who has significant changes in hematologic laboratory values or shows other evidence of visceral disease or progression too rapid to achieve benefit with radium-223 therapy. Radium-223 survival benefit has been demonstrated in the ALSYMPCA trial for administration of six treatment cycles. In patients in whom symptoms such as pain develop, supportive measures should be attempted to allow them to remain on radium-223 therapy.

\section{Sequencing therapy}

In the MDC, patients usually receive radium-223 therapy rather early in the course of their metastatic disease, when the disease burden is low with few bone metastases and no visceral metastases. Radium-223 therapy may be most effective at this point for prolonging survival and achieving the best outcome. ${ }^{5}$ Earlier use in patients with low tumor volume has the potential advantage of reducing disease burden, thus allowing more opportunity to sequence subsequent therapies during the patient's treatment course.

Patients complaining of increased bone pain or having other evidence of disease progression while receiving enzalutamide or abiraterone acetate are evaluated for radium-223 treatment. Virtually all patients initially responding to enzalutamide or abiraterone develop acquired resistance. The modest activity of these agents when used in sequence suggests a fairly high degree of cross-resistance. ${ }^{29-31}$ For example, the androgen-receptor splice variant 7 (AR-V7) is associated with resistance to both enzalutamide and abiraterone. A recent study with 31 enzalutamide-treated and 31 abiraterone-treated patients with advanced CRPC showed no PSA responses ( $\geq 50 \%$ decline) among 18 patients with AR-V7 mRNA in their tumor cells, compared with responses 
in 27 of 44 (61\%) AR-V7-negative patients. In this study, prior treatment with enzalutamide or abiraterone was associated with AR-V7 positivity. ${ }^{28}$

It is likely that there are multiple mechanisms of resistance to enzalutamide and abiraterone, and some may not be associated with cross-resistance. However, until mechanisms are defined and sensitivity can be predicted based on markers of resistance or nonresistance, substituting a new therapeutic mechanism, such as radiation or chemotherapy, after enzalutamide or abiraterone failure is more likely to produce a more favorable result than switching between these agents.

While patients who have received docetaxel chemotherapy do benefit from radium-223, the preference is to treat patients with bone-only disease with radiation therapy or radium-223 before using chemotherapy. The side effects of radium-223 are easily managed and do not preclude later chemotherapy. Patient acceptance of radium-223 is better than that of chemotherapy, as patients are typically concerned about the higher rates of myelosuppression, hair loss, hypersensitivity, nail bed changes, peripheral edema, and neuropathy experienced with taxane therapy.

\section{Radium-223 and MDC cost-effectiveness}

Evidence supports the cost-effectiveness of radium-223 treatment of patients with $\mathrm{mCRPC}$ and bone metastases in an MDC. In ALSYMPCA, the cost-effectiveness of radium-223 therapy was assessed with respect to utilization of hospitalization and other health care resources. The analysis revealed a $24 \%$ reduction in annualized hospitalization and approximately 6.5 fewer hospitalization days per patient per year among patients treated with radium-223 compared with those who received placebo. This difference was possibly driven by a delay in time to symptomatic skeletal event and reduction in hospitalization days per year after symptomatic skeletal event. There were no significant differences in terms of visits or duration of time in nursing homes, amount of adult day care and home health care services utilized, or number of physician visits. ${ }^{32}$

The cost-effectiveness of treatment received in an MDC is less clearly supported. While many clinicians believe that providing care in an MDC is inefficient and costly to their overall practice, overall survival rates for patients with advanced prostate cancer have been superior when treatment is received through an MDC. In a report on the 15-year experience in a prostate cancer MDC at the Kimmel Cancer Center of Thomas Jefferson University in Philadelphia, Gomella et $\mathrm{al}^{33}$ showed 10-year survival rates for patients with stage III and stage IV prostate cancer exceeding those of Surveillance,
Epidemiology, and End Results. The improvement was at least partially due to improved surgical procedures. It was also noted that the MDC approach reduced treatment regret through a coordinated presentation of all treatment options.

Our experience is consistent with these findings. We believe that the multidisciplinary approach fosters improved surgical outcomes with decreased surgical complications, resulting from increased surgeon experience and surgical volume, enhanced radiation oncology options, increased participation in clinical trials, and patient satisfaction. The multidisciplinary approach may also enhance the confidence of referring physicians that patients are receiving optimal therapy and may reinforce the decision to refer patients, rather than to continue managing them alone. The multidisciplinary approach reduces the likelihood that patients will be viewed as typical and treated with a standard regimen when their disease characteristics indicate an alternate approach, or in a changing treatment environment where former standards are no longer recognized as optimal. One aspect of cost-efficiency is that treating all mCRPC patients in the same manner promotes unnecessary use of newer, more costly regimens, possibly with short-term benefit. This failure may be avoided if treating physicians remain abreast of changes in standards of care as clinical studies address the complex issues around optimal sequencing of prostate cancer therapy. Outside an MDC, decisions may have less relation to patient preferences than to the specialty of the counseling physician. ${ }^{34}$ A study based on National Cancer Institute Surveillance, Epidemiology, and End Results Program data of 85,088 men with localized prostate cancer evaluated the relationship of visits to specialists and primary care physicians to treatment choice. The results showed that the physician's specialty related strongly to the treatment received, suggesting a need for more balanced information if the patient is to be included in decisions regarding treatment. ${ }^{35}$

In addition to the benefits to the patient, financial benefits to the institution can result, since patients managed in a multidisciplinary setting associated with a medical facility are more likely to be treated there throughout the course of their disease. ${ }^{33}$

Reports of patient satisfaction with multidisciplinary prostate cancer clinics are promising. ${ }^{33,36}$ In the Kimmel Cancer Center study, over $90 \%$ of patients rated the experience as good or very good and indicated that they would likely recommend the clinic to other patients. ${ }^{33}$ At MD Anderson Cancer Center in Houston, satisfaction with the process of treatment decision making was very high among patients treated in the prostate cancer MDC. In our experience, patient satisfaction is enhanced with a multidisciplinary approach; 
benefits of decreased patient anxiety and increased patient confidence are substantial and result in a preference for this approach.

\section{Summary}

Treatment of prostate cancer, particularly for patients with mCRPC, is rapidly changing for the better. Together with the increase in available options, there is a greater need for committed expertise and experience in managing individual patients and in determining the optimal sequencing of treatments over the disease course. Patients with advanced prostate cancer treated in an MDC have improved outcomes. Radium-223 therapy is ideal for illustrating the benefits of the MDC approach in optimizing patient care and successfully integrating it into an overall treatment program. The experience of the Genitourinary MDC at the The Miriam Hospital can serve as a model for other institutions.

\section{Disclosure}

Drs Renzulli and Mega have received honoraria and are consultants and speakers for Bayer HealthCare Pharmaceuticals. Ms Collins reports no conflicts of interest in this work.

\section{References}

1. Small EJ, Schellhammer PF, Higano CS, et al. Placebo-controlled phase III trial of immunologic therapy with sipuleucel-T (APC8015) in patients with metastatic, asymptomatic hormone refractory prostate cancer. J Clin Oncol. 2006;24(19):3089-3094.

2. de Bono JS, Oudard S, Ozguroglu M, et al; TROPIC Investigators. Prednisone plus cabazitaxel or mitoxantrone for metastatic castrationresistant prostate cancer progressing after docetaxel treatment: a randomised open-label trial. Lancet. 2010;376(9747):1147-1154.

3. de Bono JS, Logothetis CJ, Molina A, et al. Abiraterone and increased survival in metastatic prostate cancer. $N$ Engl J Med. 2011; 364(21):1995-2005.

4. Scher HI, Fizazi K, Saad F, et al; AFFIRM Investigators. Increased survival with enzalutamide in prostate cancer after chemotherapy. N Engl J Med. 2012;367(13):1187-1197.

5. Parker C, Nilsson S, Heinrich D, et al; ALSYMPCA Investigators. Alpha emitter radium-223 and survival in metastatic prostate cancer. N Engl J Med. 2013;369(3):213-223.

6. Rice K, Hudak J, Peay K, et al. Comprehensive quality-of-life outcomes in the setting of a multidisciplinary, equal access prostate cancer clinic. Urology. 2010;76(5):1231-1238.

7. Borsò E, Boni G, Galli L, et al. Radium 223 dichloride: a multidisciplinary approach to metastatic castration-resistant prostate cancer. Future Oncol. 2014;11(2):1-9.

8. Connell N, Mega AE, Castillo J, et al. Assessment of the effectiveness of a prechemotherapy teaching session: a Brown University Oncology Group study. J Clin Oncol. 2012;30(Suppl 34). abstr 260.

9. Xofigo (Radium Ra 223 Dichloride) Injection, for Intravenous Use [Package Insert]. Wayne, NJ: Bayer HealthCare Pharmaceuticals Inc.; 2013.

10. Metastron (Strontium-89 Chloride Injection) [Prescribing Information]. Arlington Heights, IL: Medi-Physics Inc., Amersham Healthcare; 1998.

11. Quadramet (Samarium Sm 153 Lexidronam Injection) [Prescribing Information]. Langhorne, PA: EUSA Pharma; 2009.
12. Age-dependent doses to members of the public from intake of radionuclides: Part 2. Ingestion dose coefficients. A report of a Task Group of Committee 2 of the International Commission on Radiological Protection. Ann ICRP. 1993;23(3-4):1-167.

13. Bruland OS, Nilsson S, Fisher DR, Larsen RH. High-linear energy transfer irradiation targeted to skeletal metastases by the alpha-emitter 223Ra: adjuvant or alternative to conventional modalities? Clin Cancer Res. 2006;12(20 pt 2):6250s-6257s.

14. Rubini G, Nicoletti A, Rubini D, Asabella AN. Radiometabolic treatment of bone-metastasizing cancer: from 186rhenium to 223radium. Cancer Biother Radiopharm. 2014;29(1):1-11.

15. Porter AT, McEwan AJ, Powe JE, et al. Results of a randomized phase-III trial to evaluate the efficacy of strontium- 89 adjuvant to local field external beam irradiation in the management of endocrine resistant metastatic prostate cancer. Int J Radiat Oncol Biol Phys. 1993; 25(5):805-813.

16. Oosterhof GO, Roberts JT, de Reijke TM, et al. Strontium(89) chloride versus palliative local field radiotherapy in patients with hormonal escaped prostate cancer: a phase III study of the European Organisation for Research and Treatment of Cancer, Genitourinary Group. Eur Urol. 2003;44(5):519-526.

17. Quilty PM, Kirk D, Bolger JJ, et al. A comparison of the palliative effects of strontium- 89 and external beam radiotherapy in metastatic prostate cancer. Radiother Oncol. 1994;31(1):33-40.

18. NCCN Clinical Practice Guidelines in Oncology: Prostate Cancer v2.2014; 2014. Available from: http://www.nccn.org/professionals/ physician_gls/f_guidelines_nojava.asp. Accessed October 1, 2014.

19. Basch E, Loblaw DA, Oliver TK, et al. Systemic therapy in men with metastatic castration-resistant prostate cancer: American society of clinical oncology and cancer care Ontario clinical practice guideline. J Clin Oncol. 2014;32(30):3436-3448.

20. Cookson MS, Roth BJ, Dahm P. Castration-resistant prostate cancer: AUA Guideline. American Urological Association; 2014. Available from: http://www.auanet.org/common/pdf/education/clinical-guidance/ Castration-Resistant-Prostate-Cancer.pdf. Accessed October 1, 2014.

21. Horwich A, Parker C, de Reijke T, Kataja V. Prostate cancer: ESMO clinical practice guidelines for diagnosis, treatment and follow-up. Ann Oncol. 2013;24(Suppl 6):vi106-vi114.

22. European Society for Medical Oncology (ESMO). Guidelines for the Treatment of Metastatic Castration-Resistant Prostate Cancer. European Society for Medical Oncology; 2014. Available from: http://www.esmo. org/Guidelines-Practice/Pocket-Guidelines-Mobile-App. Accessed October 1, 2014.

23. Sartor O, Coleman R, Nilsson S, et al. Effect of radium-223 dichloride on symptomatic skeletal events in patients with castrationresistant prostate cancer and bone metastases: results from a phase 3, double-blind, randomised trial. Lancet Oncol. 2014;15(7): 738-746.

24. Nilsson S, Larsen RH, Fosså SD, et al. First clinical experience with alpha-emitting radium-223 in the treatment of skeletal metastases. Clin Cancer Res. 2005;11(12):4451-4459.

25. Nilsson S, Franzén L, Parker C, et al. Bone-targeted radium-223 in symptomatic, hormone-refractory prostate cancer: a randomised, multicentre, placebo-controlled phase II study. Lancet Oncol. 2007;8(7):587-594.

26. Nilsson S, Strang P, Aksnes AK, et al. A randomized, dose-response, multicenter phase II study of radium-223 chloride for the palliation of painful bone metastases in patients with castration-resistant prostate cancer. Eur J Cancer. 2012;48(5):678-686.

27. O'Sullivan JM, Sartor O, Parker C, et al. External-beam radiation therapy (EBRT) use and safety with radium-223 dichloride (Ra) in patients (pts) with castration-resistant prostate cancer (CRPC) and symptomatic bone metastases (mets) from the ALSYMPCA trial. Ann Oncol. 2014;25(Suppl 4):iv263.

28. Antonarakis ES, Lu C, Wang $\mathrm{H}$, et al. AR-V7 and resistance to enzalutamide and abiraterone in prostate cancer. $N$ Engl J Med. 2014; 371(11):1028-1038. 
29. Badrising S, van der Noort V, van Oort IM, et al. Clinical activity and tolerability of enzalutamide (MDV3100) in patients with metastatic, castration-resistant prostate cancer who progress after docetaxel and abiraterone treatment. Cancer. 2014;120(7):968-975.

30. Noonan KL, North S, Bitting RL, Armstrong AJ, Ellard SL, Chi KN. Clinical activity of abiraterone acetate in patients with metastatic castration-resistant prostate cancer progressing after enzalutamide. Ann Oncol. 2013;24(7):1802-1807.

31. Sartor O, Gillessen S. Treatment sequencing in metastatic castrateresistant prostate cancer. Asian J Androl. 2014;16(3):426-431.

32. Cislo P, Scherer JR. Effects of radium-223 dichloride on risk for hospitalization and healthcare resource use in the phase 3 ALSYMPCA trial. J Clin Oncol. 2014;32(Suppl 30). abstr 21.
33. Gomella LG, Lin J, Hoffman-Censits J, et al. Enhancing prostate cancer care through the multidisciplinary clinic approach: a 15-year experience. J Oncol Pract. 2010;6(6):e5-e10.

34. Sommers BD, Beard CJ, D'Amico AV, Kaplan I, Richie JP, Zeckhauser RJ. Predictors of patient preferences and treatment choices for localized prostate cancer. Cancer. 2008;113(8):2058-2067.

35. Jang TL, Bekelman JE, Liu Y, et al. Physician visits prior to treatment for clinically localized prostate cancer. Arch Intern Med. 2010;170(5): 440-450.

36. Madsen LT, Craig C, Kuban D. A multidisciplinary prostate cancer clinic for newly diagnosed patients: developing the role of the advanced practice nurse. Clin J Oncol Nurs. 2009;13(3):305-309.

\section{Publish your work in this journal}

The Journal of Multidisciplinary Healthcare is an international, peerreviewed open-access journal that aims to represent and publish research in healthcare areas delivered by practitioners of different disciplines. This includes studies and reviews conducted by multidisciplinary teams as well as research which evaluates the results or conduct of such teams or healthcare processes in general. The journal covers a wide range of areas and welcomes submissions from practitioners at all levels, from all over the world. The manuscript management system is completely online and includes a very quick and fair peer-review system. Visit http://www.dovepress.com/testimonials.php to read real quotes from published authors.

Submit your manuscript here: http://www.dovepress.com/journal-of-multidisciplinary-healthcare-journal 